\title{
A PROSPECTIVE STUDY ANALYSIS OF LIMB LENGTH DISCREPANCY FOLLOWING TOTAL HIP ARTHROPLASTY
}

\author{
APPALA RAJU SANABOYINA ${ }^{1 *}$, SARASWATHI A V \\ ${ }^{1}$ Department of Orthopaedics, GITAM Institute of Medical sciences and Research, GITAM (Deemed to be University), Visakhapatnam, \\ Andhra Pradesh, India. ${ }^{2}$ Department of ENT, GITAM Institute of Medical sciences and Research, GITAM (Deemed to be University), \\ Visakhapatnam, Andhra Pradesh, India. Email: appalaraju.ortho123@gmail.com
}

Received: 18 November 2021, Revised and Accepted: 16 December 2021

\section{ABSTRACT}

Objective: The present study was designed and implemented to assess the incidence and occurrence of LLD in post-hip arthroplasty and also to evaluate the causes of limb length discrepancy both intra- and post-operative period to manage total hip arthroplasty effectively.

Methods: It is a prospective study involving 52 patients underwent for THA and conducted in the Department of Orthopedics, Tertiary Care Hospital, Visakhapatnam, India from the period of January 2013 to December 2019. The study used Southern approach or "Moore" approach. All the patients are assessed for limb length discrepancy immediately after the surgery and followed at 6 weeks, 3 months, and after 6 years.

Results: Three patients had significant limb length discrepancy among the 52 patients $(5.77 \%)$ while the remaining had no significant discrepancies in all X-rays which assess the radiological discrepancies in length. The study yields a satisfactory result as very few patients reported LLD during follow-up period and outcomes such as pain alleviation, walking capacity, limping, and patient satisfaction were insignificantly influenced by leg lengthening $(\mathrm{p} \leq 0.05)$.

Conclusion: A combination of pre-operative templating, intra-operative marking and usage of intra-operative image intensifier and an understanding of anatomy, biomechanics of hip, and implant design would reduce the error of limb length discrepancy.

Keywords: Limb length discrepancy, Total hip arthroplasty, X-ray, Clinical findings, outcome.

(C) 2022 The Authors. Published by Innovare Academic Sciences Pvt Ltd. This is an open access article under the CC BY license (http://creativecommons.org/ licenses/by/4.0/) DOI: http://dx.doi.org/10.22159/ajpcr.2022v15i2.43647. Journal homepage: https://innovareacademics.in/journals/index.php/ajpcr

\section{INTRODUCTION}

Limb length discrepancy (LLD) is an orthopedic problem in which the two legs are of unequal length. Patient's discernment of limb length inequality within a short post-operative period is quite common. Fortuitously, in most of the cases, this symptom resolves with time and physical therapy. The prevalence of LLD is generally seen in $90 \%$ cases. LLD is common in the general population, with prevalence ranging from 2.4 to $6.8 \mathrm{~mm}$ (mean magnitude of disparity is $5.2 \mathrm{~mm}$ ). In $53-75 \%$ of the population, the right leg is anatomically shorter than the left. The amount of LLD is unaffected by gender. There are discrepancies in both definitions and reported normative values due to a lack of agreement in the literature concerning a significant level of LLD and a universally acknowledged LLD assessment procedure.

Nevertheless, in few patients, there is a discernible leg length inequality and substantial disability because of tenacious aching, functional loss, abductor weakness, dysfunctional gait, and declension of conservative therapy may impose surgical interventions. In these situations, reconstruction arthroplasty is considered to be a last option; and on other hand, patient disapprobation with leg length discrepancy after total hip arthroplasty (THA) is one the most common reasons for law suits against orthopedic surgeons [1-3]. It is highly essential that the cause of LLD should be recognized before any surgical intervention is attempted. It is highly mandatory that the overall cost and the benefit should be discussed with the patient because there is no guarantee of outstanding results can be made [4]. However, in majority of the patients, minor or major LLD after THA has readily manageable symptoms. Leg length discrepancy after THA may cause some serious complications such as nerve injury including sciatic, femoral, and peroneal nerve palsy and gait abnormalities [5].

In the patients who undergoing for THA, pre-operative and postoperative limb length discrepancies are measured both clinically and radiologically [1]. Clinical measurement can be performed using a tape to measure the length of limb from ASIS (Anterior Superior Iliac Spine) to medial malleolus after squaring pelvis [6,7]. Radiological examination is through anteroposterior (AP) view of pelvis with both hips as the perpendicular distance between line joining both tear drop points medial to acetabuli and both lesser trochanters [8]. To some extent, in AP radiographs, the residual LLD below $10 \mathrm{~mm}$ is acceptable clinically, but there is no clear consent about undesirable upper limits of LLD. Although there are many pre-operative and intraoperative procedures regarding THA were explained in the available literature $[9,10]$, there is a need in the universally accepted, easy, effective and reproducible procedure, and easy to use, to minimalize the LLD after THA.

Pre-operative shortening is a common feature due to head collapse or arthritis. Limb length measurement in post-operative period was immediately performed after 6 weeks, 3 months, and after 2 years as a regular follow-up [11]. Corrections are made with heel raise immediately after discerning them and evaluated at regular followups [12]. In the present study, the author assessed the incidence and occurrence of LLD in post-hip arthroplasty and also to evaluate the causes of limb length discrepancy both intra- and post-operative period to manage total hip arthroplasty effectively.

\section{MATERIALS AND METHODS}

Study design

The present study is a prospective study conducted in the Department of Orthopedics, Tertiary Care Hospital, Visakhapatnam, India.

Study duration

The study was conducted from the period of January 2013-December 2019. 
Sample size

A total of 52 patients underwent for THA.

\section{Study subjects}

The study includes 39 males and 13 females with age ranging from 16 to 66 years and a mean of 39.2 years.

\section{Study design}

The study used Southern approach or "Moore" approach in all the cases. Informed and written consent was obtained from all patients before surgery and none of them rejected participation. All these 52 patients were follow-up for a period of 6 years after surgery.

\section{Inclusion criteria}

The following criteria were included in the study:

1. Patients of age more than 20 years and of either sex

2. X-ray of the patient's hip must show well-established arthritic changes.

\section{Exclusion criteria}

The following criteria were excluded from the study:

1. Patients $<20$ years age

2. Patients unwilling to consent for the study

3. Patients medically unfit for major surgery

4. Patients suffering with chronic illness like rheumatoid arthritis and or any active infection.

\section{METHODOLOGY}

Spinal epidural anesthesia was used in all the cases and the affected limb was draped in lateral decubitus position after thorough scrubbing. All the pressure spots are well padded and affected limb was placed with hip in $30^{\circ}$ flexion and knee $90^{\circ}$ flexion. Vertical incision was made from $6 \mathrm{~cm}$ distal to tip of greater trochanter (GT) to $5 \mathrm{~cm}$ proximal to GT. Skin and fat layers were incised, fascia lata, iliotibial band, and the outer fascia of gluteus maximus were incised and fibers of gluteus maximus split along the fibers proximally. Distally, the insertion of gluteus maximus was incised by cautery from fascia lata to relax sciatic nerve. The short external rotators were identified and detached from their insertion as a separate layer. A skin suture of fixed length of ethibond 5- 0 just below the iliac crest and with the hip and knee in a fixed plane of $30^{\circ}$ and in neutral coronal plane marking was done over the greater trochanter with diathermy, which was used at a later stage to check intra-operative lengthening. Capsule was incised as a separate layer and hip dislocated with hip in flexion, adduction, and internal rotation. Femoral neck osteotomy is made with an oscillating saw proximal to lesser trochanter based on pre-operative templating. Acetabulam was exposed with help of two retractors; one placed over anterior column and other near transverse acetabular ligament, a Steinmann pin was used to retract the capsule posteriorly and pinned over the ischium to have a $360^{\circ}$ view of acetabulam. Acetabulam was reamed and press fit was achieved with trial till the true floor was exposed. In 48 cases, uncemented press fit arthroplasty was used and fixed the acetabulam with screw, and in four cases, cemented arthroplasty was used. Femoral trial was performed with trial implants after acetabulam was fixed with liner. After routine broaching and trial fixation, the length was rechecked and soft-tissue tension of capsule and short rotators assessed along with skin suture. After the confirmation of desired length, the original implant was inserted. Capsule was closed as a separate layer with ethibond and soft tissues are closed with vicryl and skin staples were applied.

The content report forms regarding the LLD were filled by the surgeons which include perioperative information about patient history, clinical examination, and surgical intervention. Moreover, in the similar manner, clinical outcomes such as walking capacity ( $>60 \mathrm{~min}, 31-60 \mathrm{~min}$, 13-30 min, and <10 min), hip pain (none, mild, moderate, severe, and intolerable), limp without support (none, slight, moderate, and severe), and patient satisfaction (excellent, good, fair, and poor) were recorded during follow-up examinations.
The HHS was used for the assessment of the results of THA and is used to evaluate the various hip disabilities and methods of the treatment in the present study population. The domains in HHS include pain, function, absence of deformity, and range of motion. The pain domain measures pain severity and its effect on activities and needs for pain medication. The function domain contains of daily activities (stair use, using public transportation, sitting, and managing shoes and socks) and gait (limp, support needed, and walking distance). Deformity takes into account hip flexion, adduction, internal rotation, and extremity length discrepancy. Range of motion measures hip flexion, abduction, external and internal rotation, and adduction. There are ten items in HHS. The score has a maximum of 100 points (best possible outcome) covering pain (one item, 0-44 points), function (seven items, $0-47$ points), absence of deformity (one item, four points), and range of motion (two items, five points). The HHS score gives a maximum of 100 points. Pain receives 44 points, function 47 points, range of motion five points, and deformity four points. Function is subdivided into activities of daily living (14 points) and gait (33 points).

\section{RESULTS}

All the patients are assessed for limb length discrepancy immediately after the surgery and followed at 6 weeks, 3 months, and after 6 years. Three patients had significant limb length discrepancy among the 52 patients $(5.77 \%)$, while the remaining had no significant discrepancies (Fig. 1). Transverse lines were drawn connecting hip centers, inferior part of tear drops, and lesser trochanters for all X-rays to assess the radiological discrepancies in length. Lengthening was seen as a compliant in two persons $1.5 \mathrm{~cm}$ and $1 \mathrm{~cm}(3.8 \%)$ requiring heel raise on contra lateral side (Fig. 2).One patient had shortening in followup period though her immediate post-operative period was normal due to subsidence of femoral stem of $1 \mathrm{~cm}$ (Figs. 3 and 4). The patient had

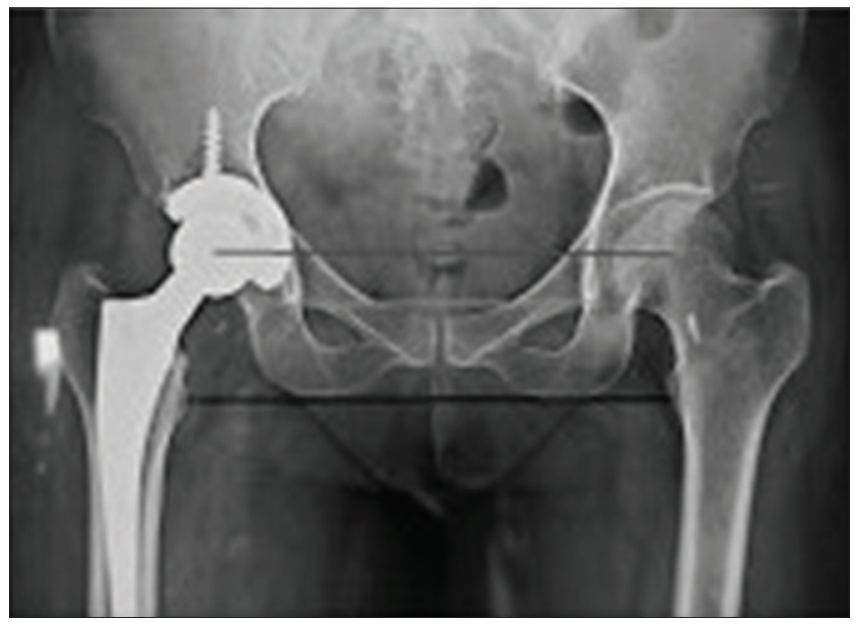

Fig. 1: Equal limb length

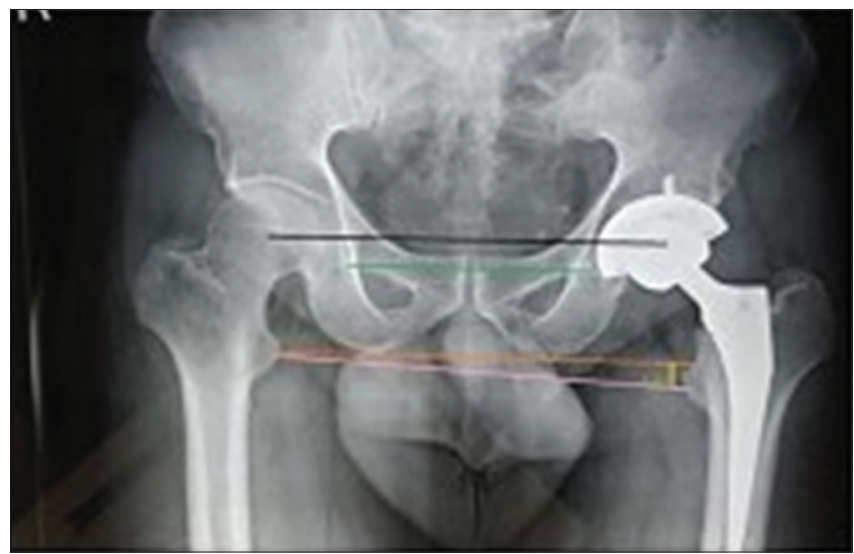

Fig. 2: Lengthened by $1 \mathrm{~cm}$ 


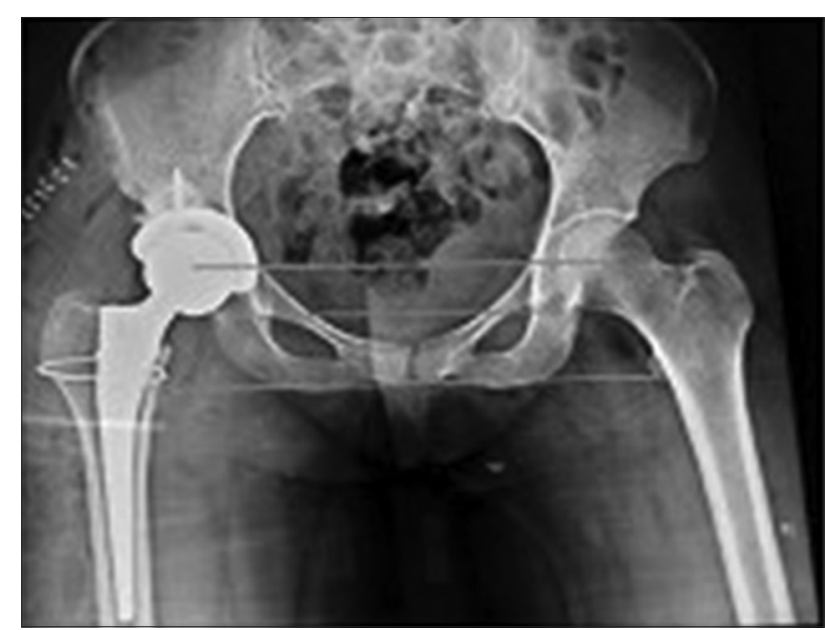

Fig. 3: Immediate post-operative period

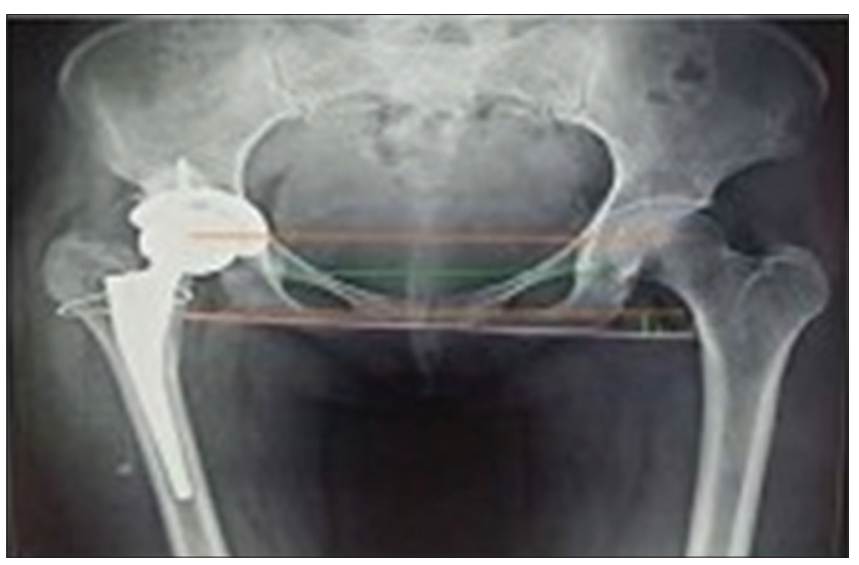

Fig. 4: Subsidence

presented with limp at 3 weeks due to subsidence of stem and was managed with protected weight bearing and after 6 months the limp settled down and the patient was managed with a shoe raise of $1 \mathrm{~cm}$ on the affected side. From the results, it was observed that the study yields satisfactory outcomes as very few patients reported LLD during followup period and outcomes such as pain alleviation, walking capacity, limping, and patient satisfaction were insignificantly influenced by leg lengthening ( $\mathrm{p} \leq .0 .05)$. Harris hip score was 76 at 6 weeks due to subsidence and improved to 93 at 3 months and at 2 years followup after osseointegration in patient with shortening. The higher the HHS, the less dysfunction of limb. A total score of $<70$ is considered a poor result; $70-80$ is considered fair, $80-90$ is good, and $90-100$ is an excellent result. Harris hip score was 89 in both patients with $1 \mathrm{~cm}$ and $1.5 \mathrm{~cm}$ lengthening at 3 months. The scores remained same even at 2 years and shortening was accepted better than lengthening based on Harris hip scoring done by the surgeon. While the mean post-operative Harris hip score was 96.8 in the remaining patients at 2 years follow-up and HHS was excellent in majority of the cases.

\section{DISCUSSION}

Limb length discrepancy (LLD) is an orthopedic problem in which the two legs are of unequal length after THA and it is not a rare problem; and in most cases, the condition will progress with time period and good physical therapy. However, limb length discrepancy is an allied litigious problem involving with patient dissatisfaction and poorer outcome after THA against orthopedic surgeons [13]. The most common symptoms include pain, paresthesias, and instability of gait. After an extensive literature review, it is observed that the percentage of limb length discrepancy varies from $1 \%$ to $27 \%$. In the present study, out of 52 patients who underwent THA surgery, three patients had significant limb length discrepancy among the 52 patients (5.77\%) while the remaining had no significant discrepancies. Lengthening was seen as a compliant in two persons $1.5 \mathrm{~cm}$ and $1 \mathrm{~cm}(3.8 \%)$ requiring heel raise on contra lateral side and one patient had shortening limb after post-operative period and was normal due to subsidence of femoral stem. The study yielded an excellent satisfactory results as very few cases were reported with LLD and the prevalence of LLD showed in the present investigation was in accordance with the results reported by the Ranawat and Rodriguez [12] who studied a series of patients with functional limb length discrepancy and showed that it resolved in all cases by 6 months with proper physical therapy, despite an initial high prevalence. The present study results were deviated from the report of Konyves and Bannister [13] who assessed 90 patients who underwent THA. After THA, the authors identified 56 (62\%) cases have long limb length and during 3 month follow-up period, the LLD cases were reduced to 24 (43\%); and later on after 12 month followup period, the LLD cases were further reduced to 18 (33\%). Sudhahar et al. [14] in their study showed subsidence of uncollared corail stem in $28 \%$ up to $3 \mathrm{~mm}$ and $7 \%$ subsidence in collared stems. The authors agreed that collared stems should also be used as a standby in case of suspicion of weak calcar which requires further studies to support. Roder et al. [15] identified 478 LLD cases with post-operative lengthening and 275 with shortening of limb length during 10415 regular follow-up examinations. They concluded that walking capacity, limping, and patient satisfaction were all significantly associated with leg lengthening, whereas pain alleviation was not. In contrast, hip pain, limping and patient satisfactions were all significantly associated with leg shortening, whereas walking capacity was not. Sanjay Kumar et al. [16] noticed shorter limb length as compared to contra lateral side during the pre-operative period and during post-operative period; the lengthening of limb was identified as $42.9 \%(9 / 21)$ and $19 \%(4 / 21)$ of cases shortening of limb was identified and in 31\% (8/21) postoperative limb lengths were equal. Functional scores were excellent in patients with equal limb length as compared to those having shorter or longer limb length. The prevalence of LLD was higher compared to the results of the present study. Clinically, equal limb length was accompanied with the finest functional results and highest satisfaction rates. A shorter operated leg was involved with limping and longer operated leg had more hip pain. The increased pain causes patient dissatisfaction, but the detected difference in pain can be attributed to the limb length discrepancy.

\section{CONCLUSION}

Although limb length discrepancy is a known complication of total hip arthroplasty, efforts should be made to reduce the incidence of length inequality for better gait pattern, post-operative hip scores, functional outcome, and patient satisfaction. A combination of pre-operative templating, intra-operative marking and usage of intra-operative image intensifier and an understanding of anatomy, biomechanics of hip, and implant design would reduce the error of limb length discrepancy.

\section{ACKNOWLEDGMENT}

The authors would like to thank all the faculty members and technicians for their support during the period of study.

\section{DECLARATION OF PATIENT CONSENT}

The author certifies that all appropriate consent forms have been obtained from the patients' parents to publish the article. In the forms, they have given their consent for images and other clinical information to be reported in the journal. They have understood that the patients' names and initials will not be published and due efforts will be made to conceal the patients' identity, but anonymity cannot be guaranteed.

\section{CONFLICT OF INTEREST}

The authors declared no conflict of interest 


\section{AUTHORS' CONTRIBUTION}

The main author of the study ARS had performed the work and wrote the article. The author SAV collected the literature.

\section{SOURCE(S) OF SUPPORT (IF ANY)}

The study was not supported by any grants and funds.

\section{ETHICS CLEARANCE}

A proposal regarding the study's aims and objectives was submitted to the Institutional Ethics Committee, GITAM Deemed to be University, Visakhapatnam and permission was obtained from the Institutional Ethics Committee regarding data collection.

\section{REFERENCES}

1. Parvizi J, Sharkey PF, Bissett GA, Rothman RH, Hozack WJ. Surgical treatment of limb-length discrepancy following total hip arthroplasty. J Bone Joint Surg Am 2003;85:2310-7.

2. Hofmann AA, Skrzynski MC. Leg-length inequality and nerve palsy in total hip arthroplasty: A lawyer awaits! Orthopedics 2000;23:943-4.

3. Meermans G, Malik A, Witt J, Haddad F. Preoperative radiographic assessment of limb-length discrepancy in total hip arthroplasty. Clin Orthop Relat Res 2011;469:1677-82.

4. Abraham WD, Dimon JH. Leg length discrepancy in total hip arthroplasty. Orthop Clin North Am 1992;23:201-9.

5. Ranawat CS, Rao RR, Rodriguez JA, Bhende HS. Correction of limb length inequality during total hip arthroplasty. J Arthroplasty
2001;6:715-20.

6. Sayed-Noor AS, Hugo A, Sjoden GO, Wretenberg P. Leg length discrepancy in total hip arthroplasty: Comparison of two methods of measurement. Int Orthop 2009;33:1189-93.

7. Moretti VM, Zachary DP. Surgical approaches for total hip arthroplasty. Indian J Orthop 2017;51:368-76.

8. Chechik O, Khashan M, Lador R, Salai M, Amar E. Surgical approach and prosthesis fixation in hip arthroplasty worldwide. Arch Orthop Trauma Surg 2013;133:1595-600.

9. Clark CR, Huddleston HD, Schoch EP, Thomas BJ. Leg-length discrepancy after total hip arthroplasty. J Am AcadOrthop Surg 2006; 14:38-45.

10. Hossain M, Sinha AK. A technique to avoid leg-length discrepancy in total hip arthroplasty. Ann R Coll Surg 2007;89:314-5.

11. Desai AS, Dramis A, Board TN. Leg length discrepancy after total hip arthroplasty: A review of literature. Curr Rev Musculoskelet Med 2013;6:336-41.

12. Ranawat CS, Rodriguez JA. Functional leg-length inequality following total hip arthroplasty. J Arthroplasty 1997;12:359-64.

13. Konyves A, Bannister GC. The importance of leg length discrepancy after total hip arthroplasty. J Bone Joint Surg 2005;87-B:155-7.

14. Sudhahar TA, Morapudi S, Barnes K. Evaluation of subsidence between collarless and collared Corail femoral cementless total hip replacement. J Orthop 2009;6:e3.

15. Röder C, Vogel R, Burri L, Dietrich D, Staub LP. Total hip arthroplasty: Leg length inequality impairs functional outcomes and patient satisfaction. BMC Musculoskelet Disord 2012;13:95.

16. Kumar S, Kumar A, Bharti A, Gupta AK, Senger GK. Analysis of the functional outcome of limb length discrepancy after total hip replacement. Annals of International Med Dent Res 2017;4:1-7. 